\title{
Comparison of sodium tetradecyl sulfate, bleomycin, and talc powder for rat pleurodesis induction
}

\author{
Mohammadreza Lashkarizadeh ${ }^{1}$ (1) $\cdot$ Mohammad Rezazadehkermani $^{1} \cdot$ Hadi Hadavi $^{1} \cdot$ Mahdieh Lashkarizadeh $^{2} \cdot$ \\ Razieh Dehghanfiroozabadi ${ }^{1}$
}

Received: 5 December 2018 / Accepted: 16 August 2019/Published online: 4 September 2019

(C) The Author(s) 2019

\begin{abstract}
Malignant pleural effusion greatly alters patient's quality of life and pleurodesis significantly improves this index in these patients. Numerous agents have been introduced for pleurodesis. In this study, sodium tetradecyl sulfate (STS) is used for induction of pleurodesis and its efficacy is compared with other sclerosing agents: talc powder and bleomycin. Randomly divided four groups of rats undergone pleurodesis using STS, bleomycin, talc powder, and normal saline. The efficacy of each agent is investigated both microscopic and grossly. The most severe fibrosis was induced by bleomycin and STS respectively and this fibrosis was statistically significant in microscopic evaluation scales. Grossly the most severe fibrosis was induced by bleomycin. Talc Powder and STS caused less fibrosis but the same degree in gross evaluation. This study demonstrates the efficacy of STS for induction of pleurodesis in rat; however, further studies should be done for evaluation of its safety and efficacy in human subjects.
\end{abstract}

Keywords Sodium tetradecyl sulfate $\cdot$ Talc $\cdot$ Bleomycin $\cdot$ Pleurodesis

\section{Introduction}

Pleurodesis is defined in last decades to attach the visceral and parietal pleura in patients with malignant and non-malignant conditions. The procedure is not therapeutic but improves

Mahdieh Lashkarizadeh

mh.lashkarizadeh@gmail.com

Mohammadreza Lashkarizadeh

lashkarizadeh@kmu.ac.ir

Mohammad Rezazadehkermani

rezazadehkermani@yahoo.com

Hadi Hadavi

hadihadavi.md@gmail.com

Razieh Dehghanfiroozabadi

meesmaeel-panjaalizade@yahoo.com

1 Department of General Surgery, School of Medicine, Kerman University of Medical Sciences, Kerman, Iran

2 Department of Pathology and Stem Cell Research Center, School of Medicine, Kerman University of Medical Sciences, Kerman, Iran patient's quality of life by decreasing dyspnea. The main mechanism is chemical pleuritis and subsequent fibrosis. Failure rate of pleurodesis is different with sclerosing agents and some indices have been described for predicting successful pleurodesis. (Light 2013)

Numerous agents have been used for induction of pleurodesis such as talc powder, bleomycin, iodine povidone, autologous blood, tetracycline, and many other agents. Each agent has its own advantage and disadvantages and success rate of pleurodesis is different with each agent. (Suárez and Gilart 2013)

There are some complications that are reported with pleurodesis including acute respiratory distress syndrome (Light 2013) and activation of systemic coagulation and thrombotic events. (Rodriguez-Panadero and MontesWorboys 2012) This issue demonstrates the necessity for searching newer sclerosing agents for safer pleurodesis.

Sodium tetradecyl sulfate (STS) is a detergent substance that works by disrupting the membrane of cells. It was approved by the Food and Drug Administration since 1946 for sclerotherapy of varicose veins. (Jenkinson et al. 2017) Rather than venous sclerotherapy, it is used for treatment of hydrocele (Musa et al. 2015), renal cyst (Demir et al. 2007), and Kaposi sarcoma (Ramírez-Amador et al. 2002). 
Fig. 1 Fibrosis scale with hematoxylin and eosin staining technique (a-d). a Mild fibrosis. b Sever fibrosis. c No fibrosis. d Moderate fibrosis

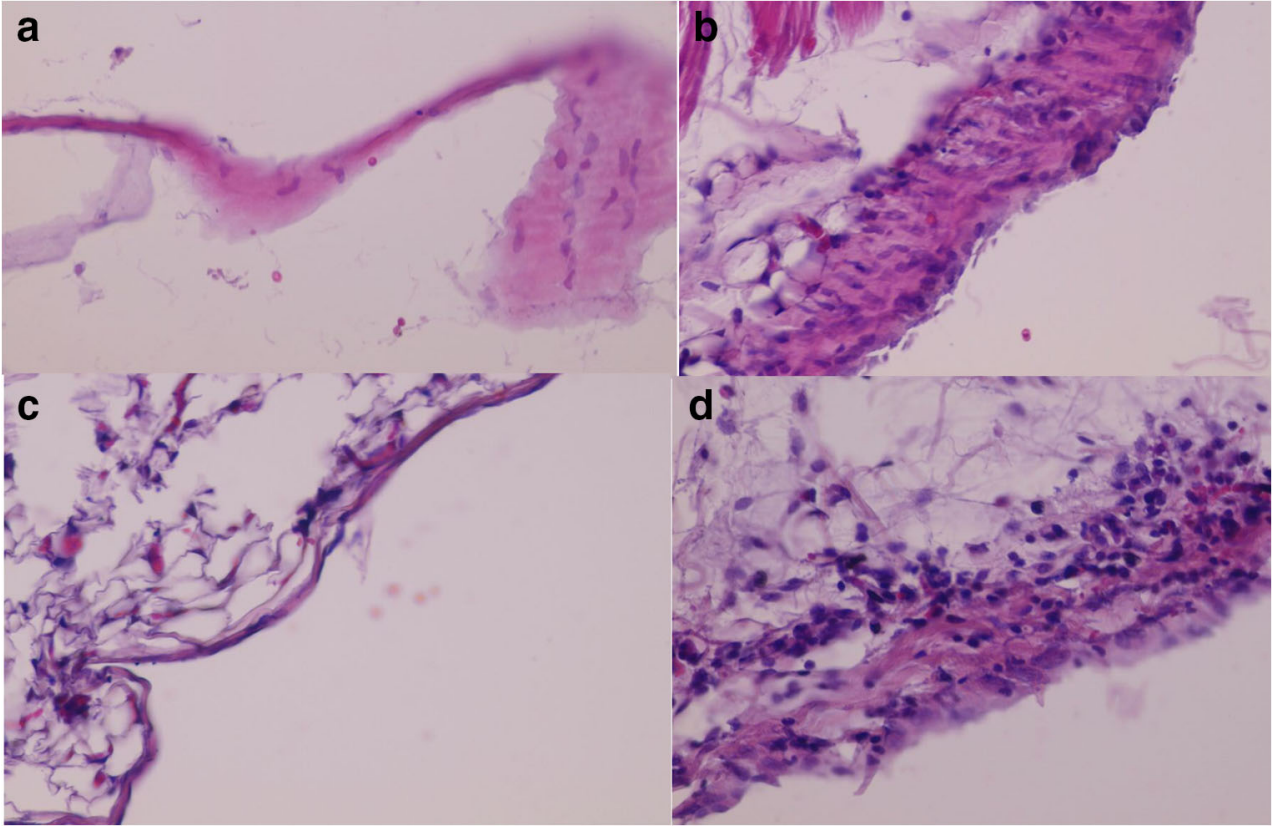

In this study, the authors introduce STS as a previously known sclerosing agent as a pleurodesis agent and compare its efficacy in animal model of pleurodesis with other agents.

\section{Material and methods}

After approval of the ethics committee at Kerman University of Medical Sciences regarding guide lines for care and use of laboratory animals, the study was started at Kerman Neuroscience Research Center animal lab.

Adult male rats with mean weight of $270 \mathrm{~g}$ (225$320 \mathrm{~g}$ ) were enrolled in the study. These rats were kept in standard cages with free access to the water and food, physiologic day night cycles and constant temperature and humidity.

The rats were randomly assigned to four groups as STS, bleomycin, talc, and normal saline. Each group comprised nine rats. All of the animals were anesthetized with combination of ketamine and xylazine. Under general anesthesia with aseptic condition, a 1-cm incision was made over the seventh rib at the level of posterior axillary line. After creating a subcutaneous

Table 1 Fibrosis severity between groups

\begin{tabular}{llllll}
\hline Fibrosis & STS & Saline & Talc & Bleomycin & $P$ value \\
\hline Mild & $3(33.33 \%)$ & $8(88.8 \%)$ & $3(33.3 \%)$ & 0 & \\
Moderate & $3(33.33 \%)$ & $1(12.2 \%)$ & $5(55.5 \%)$ & $2(30 \%)$ & 0.017 \\
Severe & $3(33.33 \%)$ & $0(\%)$ & $1(12.2 \%)$ & $7(70 \%)$ & \\
\hline
\end{tabular}

tunnel, a 5-French catheter was introduced in to the right pleural space via this tunnel. The sclerosing agent was injected via the catheter and after aspiration of excess air the catheter was removed.

The sclerosing agents were diluted in $0.5 \mathrm{cc}$ of normal saline. The final concentration of STS was $1 \%$ and the concentrations of bleomycin ( 5 unit $/ \mathrm{kg}$ ) and talc $(60 \mathrm{mg} / \mathrm{kg}$ ) were adjusted regarding animal weight.

After 30 days, the animals were euthanized and the lungs with overlying chest wall were completely excised. The lungs were expanded with intratracheal injection of formalin and the specimen was kept in buffer solution for $48 \mathrm{~h}$.

After routine histopathology tissue processing sections were provided with $5 \mu \mathrm{m}$ thickness, Masson trichrome staining was used on visceral pleura sections and slides were reviewed with two independent pathologists with 10 years academic experience.

The samples were investigated both microscopic and grossly. Regarding previous reports (Mitchem et al. 1999), the specimens were grossly graded with following scale: 0 considered normal, 1 as scattered point fibrosis, 2 as diffused adhesion, and 3 as complete obliterating of pleural space with adhesion.

After standard hematoxylin and eosin staining, the cut sections were investigated for inflammation and cellularity and graded as follows: 1, few collagen fibers (mild); 2, more collagen fibers but not well organized (moderate); 3 , high-density collagen fibers partially organized in thick layers (severe). (Hashemzadeh et al. 2012)

Gathered data were analyzed multivariate Fisher exact test using SPSS software. 


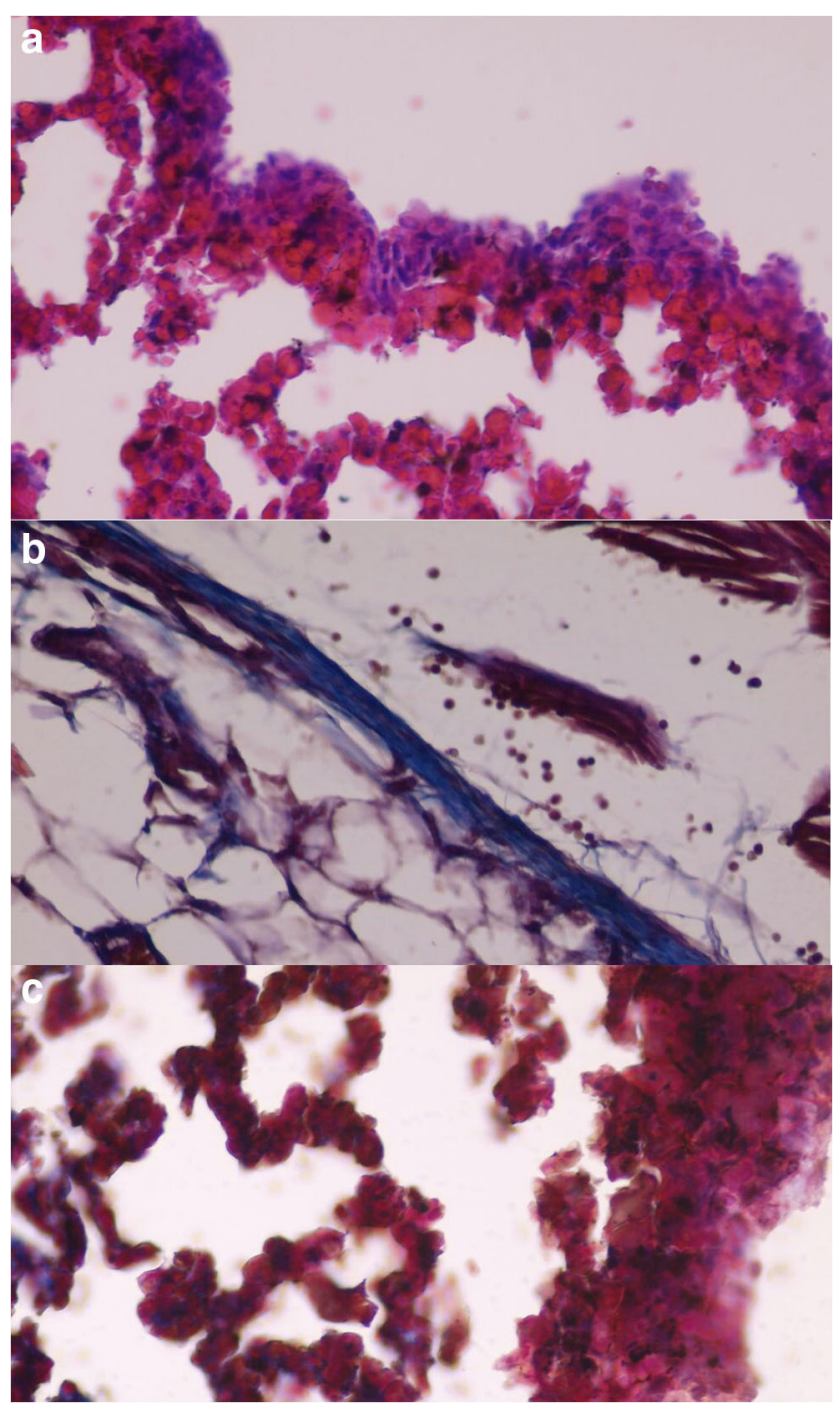

Fig. 2 Fibrosis scale with Masson trichrome staining technique (a-c). a Mild fibrosis. b Moderate fibrosis. c No fibrosis

\section{Results}

The most severe fibrosis in this study was induced by bleomycin, STS, and talc respectively. Figure 1 reveals fibrosis evaluation using hematoxylin and eosin staining. Moderate fibrosis was more prevalent in the talc group. Saline injection in to the pleural cavity was associated with mild fibrosis in eight specimens out of nine. Table 1 demonstrates the distribution of fibrosis severity between different groups. Figure 2

Table 2 Inflammation severity between groups

\begin{tabular}{llllll}
\hline Inflammation & STS & Saline & Talc & Bleomycin & $P$ value \\
\hline Mild & 0 & $6(60 \%)$ & $3(30 \%)$ & $1(10 \%)$ & \\
Moderate & 0 & 0 & $2(25 \%)$ & $6(75 \%)$ & 0.034 \\
Severe & $6(85.6 \%)$ & 0 & $1(14.3 \%)$ & 0 & \\
\hline
\end{tabular}

Table 3 Mesothelial hyperplasia in different groups

\begin{tabular}{llllll}
\hline Hyperplasia & STS & Saline & Talc & Bleomycin & $P$ value \\
\hline Mild & 0 & 0 & $3(30 \%)$ & $7(70 \%)$ & 0.024 \\
Severe & $6(42.9 \%)$ & $8(57.1 \%)$ & 0 & 0 & \\
\hline
\end{tabular}

demonstrates fibrosis scale with Masson trichrome staining technique.

Table 2 demonstrates the inflammation severity in different groups. It shows that STS caused higher degree of inflammation rather than Talc and bleomycin and this difference is statistically significant. Comparing groups in Tables 1 and 2, it is seen that moderate inflammation was associated with more severe fibrosis.

Mesothelial hyperplasia was evaluated in the specimens. Mild degree of mesothelial hyperplasia was found in talc and bleomycin groups, but severe mesothelial hyperplasia was seen in STS and normal saline groups which is not related to the degree of inflammation or fibrosis. Table 3 shows the distribution between different groups.

Gross evaluation of animals' thoracic cavity showed the greatest amount of fibrosis in the bleomycin group while talc and STS induce the same amount of fibrosis and both were less than bleomycin. This difference was also statistically significant (Table 4).

No mortality was encountered during the study and one case of lung fibrosis was seen in the STS group. Lung alveolar hemorrhage (Fig. 3) was reported in one of the animals who received talc.

\section{Discussion}

This study investigates the efficacy of STS in pleurodesis induction. Compared with known pleurodesis agents such as talc powder and bleomycin, it induced greater pleurodesis markers such as inflammation and mesothelial hyperplasia. Fibrosis severity in the STS pleurodesis group was greater than Talc and less than bleomycin while gross fibrosis was the same as talc powder and less than bleomycin.

This report demonstrates the high efficacy of bleomycin in pleurodesis induction but its clinical use is limited due to high cost of drug (MC et al. 2006) and risk of systemic absorption and possible toxicity. (Rodriguez-Panadero and Antony 1997)

Table 4 Gross fibrosis evaluation in different groups

\begin{tabular}{llllll}
\hline Fibrosis & STS & Saline & Talc & Bleomycin & $P$ value \\
\hline Negative & $3(33.33 \%)$ & $8(88.8 \%)$ & $3(33.33 \%)$ & 0 & 0.003 \\
Positive & $6(66.66 \%)$ & $1(12.2 \%)$ & $6(66.66 \%)$ & $9(100 \%)$ & \\
\hline
\end{tabular}




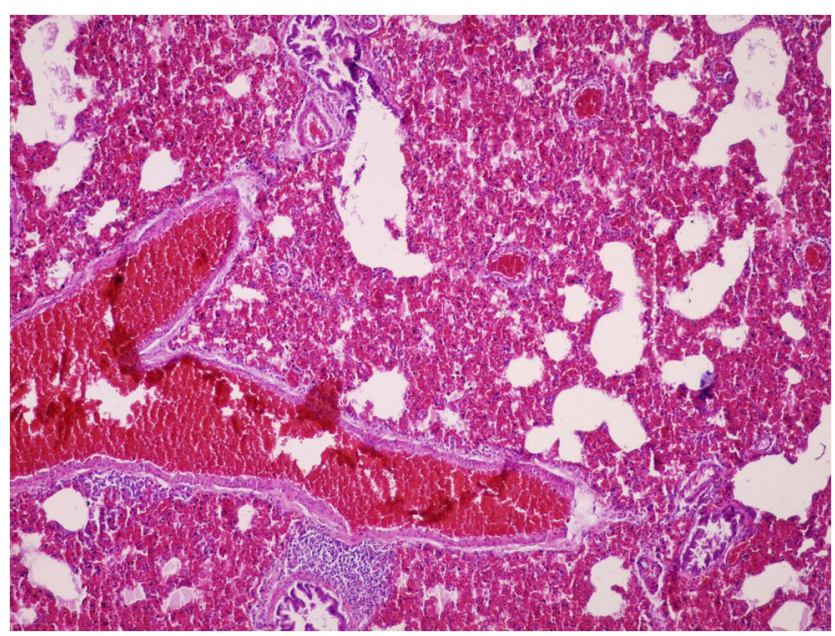

Fig. 3 Alveolar hemorrhage in talc pleurodesis group

STS is a known sclerosing agent commonly used in sclerotherapy of varicose veins (Jenkinson et al. 2017) and in literature review despite reports about using it for sclerosing of different organs, there were no reports about using STS in pleurodesis. It is thought that detergent sclerosants such as STS cause total endothelial destruction results in subendothelial exposure which leads to exposure of collagen fibers (Duffy 2010) with presumption that the same process might occur by intrapleural injection of STS the study was designed.

Severe mesothelial damage is the key element in acceptable pleurodesis. With induction of pleurodesis lots of inflammatory mediators is released from mesothelium. After the first stages of inflammation, fibroblasts have the paramount role in pleurodesis induction and it is proven that tetracyclines and talc cause fibroblast growth factor release by mesothelium. (Rodriguez-Panadero and Antony 1997)

We found the greatest mesothelial hyperplasia in the STS group which might be due to greater irritation of mesothelium by STS but gross fibrosis which is the main outcome of pleurodesis was less than bleomycin and the same as talc powder. We investigate pleural cavity 30 days after pleurodesis which is the same period as other studies (Hashemzadeh et al. 2012) so the possibility of missing pleurodesis due to early investigation of thoracic cavity is less probable. However, previous studies did not use STS and the nature of STS-induced inflammation may be different.

Although our study showed the potency of STS for pleurodesis induction in animal model, further studies are suggested for evaluation of inflammation and fibrosis in longer periods. Since intrapleural injection of STS is not investigated before, it is suggested to evaluate its complication regarding injection into the pleural space.
Funding information This study was financially supported by a grant from Kerman University of Medical Science Vice Research Chancellor.

Compliance with ethical standards After approval of the ethics committee at Kerman University of Medical Sciences regarding guide lines for care and use of laboratory animals, the study was started at Kerman Neuroscience Research Center animal lab.

Conflict of interest The authors declare that there is no conflict of interest.

Open Access This article is distributed under the terms of the Creative Commons Attribution 4.0 International License (http:// creativecommons.org/licenses/by/4.0/), which permits unrestricted use, distribution, and reproduction in any medium, provided you give appropriate credit to the original author(s) and the source, provide a link to the Creative Commons license, and indicate if changes were made.

\section{References}

Demir E, Alan C, Kilciler M, Bedir S (2007) Comparison of ethanol and sodium tetradecyl sulfate in the sclerotherapy of renal cyst. J Endourol 21(8):903-905

Duffy D (2010) Sclerosants: a comparative review. Dermatol Surg 36(Suppl 2):1010-1025

Hashemzadeh S, Hashemzadeh K, Mamaghani K, Ansari E, Aligholipour R, Golzari SE et al (2012) Pleurodesis by erythromycin, tetracycline, AerosilTM 200, and erythromycin plus AerosilTM 200 in a rat model: a preliminary study. DARU J Pharm Sci 20:79

Jenkinson HA, Wilmas KM, Silapunt S (2017) Sodium tetradecyl sulfate: a review of clinical uses. Dermatol Surg 43:1313-1320

Light RW (2013) Pleural diseases, 6th edn. Lippincott Williams \& Wilkins, Philadelphia

Mitchem RE, Herndon BL, Fiorella RM, Molteni A, Battie CN, Reisz GR (1999) Pleurodesis by autologous blood, doxycycline, and talc in a rabbit model. Ann Thorac Surg 67:917-921

Musa O, Roy A, Ansari N, Sharan J (2015) Evaluation of the role of sodium tetradecyl sulfate as a sclerosant in the treatment of primary hydrocele. Indian J Surg 77(Suppl 2):432-437

Ramírez-Amador V, Esquivel-Pedraza L, Lozada-Nur F, De la RosaGarcía E, Volkow-Fernández P, Súchil-Bernal L et al (2002) Intralesional vinblastine vs. $3 \%$ sodium tetradecyl sulfate for the treatment of oral Kaposi's sarcoma. A double blind, randomized clinical trial. Oral Oncol 38(5):460-467

Rodriguez-Panadero F, Antony VB (1997) Pleurodesis: state of the art. Eur Respir J 10:1648-1654

Rodriguez-Panadero F, Montes-Worboys A (2012) Mechanisms of pleurodesis. Respiration. 83:91-98

Suárez PMR, Gilart JLF (2013) Pleurodesis in the treatment of pneumothorax and pleural effusion. Monaldi Arch Chest Dis 79(2):81-86

Vaz MC, Marchi E, Vargas FS (2006) Pleurodesis: technique and indications. J Bras Pneumol 32(4):347-356

Publisher's note Springer Nature remains neutral with regard to jurisdictional claims in published maps and institutional affiliations. 